\title{
Profilaxis de toxoplasmosis en niños y adultos sometidos a trasplante de órganos sólidos y precursores hematopoyéticos
}

\author{
Ernesto Payá, Isabel Noemí, Renzo Tassara, Paula Catalán y Carmen L. Avilés
}

\section{Prophylaxis against Toxoplasma gondii disease in pediatric and adult patients undergoing solid organ and hematopoietic stem cells transplantation}

Toxoplasmosis is a widely distributed zoonosis produced by the parasite $T$. gondii. In Chile the seroprevalence has been estimated between $20-37 \%$ in general population. Defined risk groups acquire or reactivate the infection by $T$. gondii in patients undergoing SOT and HSCT are: heart transplant or heart-lung with D (+) and R (-), allogeneic HSCT with R (+), HSCT with cord cells, GVHD, history of previous clinical toxoplasmosis and use of corticosteroids for prolonged periods or in high doses. Hand washing, hygiene in food handling and weekly post-transplant surveillance since day 15 post transplant for six months, are universally recommended. All patients with SOT and HSCT, regardless of risk, should receive prophylaxis with cotrimoxazole and require no another specific prophylaxis against $T$. gondii (A2). It is particularly important in high-risk patients who cannot receive cotrimoxazole prophylaxis establish specific alternative against $T$. gondii (B3).

Key words: Toxoplasmosis, Toxoplasma gondii, prophylaxis, transplant, solid organ transplantation, hematopoietic stem cells transplantation.

Palabras clave: Toxoplasmosis, Toxoplasma gondii, profilaxis, trasplante, trasplante de órganos sólidos, trasplante de precursores hematopoyéticos.

\section{Introducción}

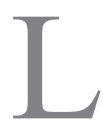

a toxoplasmosis es una zoonosis ampliamente distribuida, producida por el parásito T. gondii. Según la Organización Mundial de la Salud (2006), existen 7,5 millones de infectados y 55.185 nuevos casos por año. En América hay 80 a 100 millones de personas en riesgo de adquirir la enfermedad. En los pacientes inmunocomprometidos se presenta habitualmente como una encefalitis, caracterizada clínicamente por cefalea progresiva, confusión, ataxia, convulsiones y finalmente coma. Otras formas de presentación son neumonía, miocarditis y toxoplasmosis diseminada.

\section{Epidemiología}

En Chile se ha definido una seroprevalencia en la población general de $37 \%$ en $1996^{1}$ y $25 \%$ en $1997^{2}$, y específicamente en la Región de Los Lagos se ha definido una seroprevalencia de $20 \%$ en $1998^{3}$, no existiendo otros estudios nacionales actualizados. En Europa Central la seroprevalencia es de 50 a 80\%, en Japón de 10 a 15\% y en E.U.A. de $15 \%$.

La incidencia de toxoplasmosis en trasplante de órganos sólidos (TOS) es mayor en receptores seronegativos de trasplante cardíaco con donante seropositivo (50 a $70 \%$ de toxoplasmosis clínica en series sin profilaxis $)^{4,5}$ y menor en receptores de TOS no cardíaco ${ }^{6,7}$.
En receptores de trasplante de precursores hematopoyéticos (TPH) en el concierto internacional, esta seroprevalencia varía de 10 a $71 \%$ y está en estrecha relación con la prevalencia en la población general. La enfermedad clínica oscila entre 0,1 y $6 \%$ en los receptores seropositivos con 60 a $90 \%$ de mortalidad.

\section{Factores de riesgo}

Todos los pacientes sometidos a TOS o TPH están en riesgo de contraer o de reactivar una toxoplasmosis ${ }^{178}$. Sin embargo, dentro de esta población, existe una clara diferencia en el riesgo dependiendo del tipo de trasplante:

- Pacientes de menor riesgo: pacientes con TOS no cardíaco, TPH autólogo, TPH alogeneicos con R (-), trasplante de tejido ocular, óseo o vascular, TOS con D y R (+) o D (-) 4 . Los pacientes seronegativos tienen el riesgo potencial de adquirir la enfermedad con una evolución de mayor gravedad que los seropositivos, por lo que las medidas de prevención no farmacológicas son vitales en ellos 5 .

- Pacientes de mayor riesgo: los sometidos a trasplante cardíaco o de corazón-pulmón con D (+) y R (-); TPH alogénico con $\mathrm{R}(+)$; TPH con células de cordón; EICH; antecedentes de toxoplasmosis clínica ${ }^{4}$ y uso de corticoesteroides por tiempo prolongado ( $>$ a 14 días) o en altas dosis ( $\geq 1 \mathrm{mg} / \mathrm{kg} /$ día de metilprednisolona o equivalentes).
Servicio de Pediatría, Hospital de Carabineros. Universidad Mayor, Santiago (EP).

Departamento de Pediatría, Hospital Dr. Luis Calvo Mackenna, Santiago. Facultad de Medicina, Universidad de Chile (IN).

Departamento de Pediatría, Hospital San Juan de Dios, Santiago. Facultad de Medicina, Universidad de Chile (RT).

Unidad de Trasplante de Precursores Hematopoyéticos, Hospital Dr. Luis Calvo Mackenna, Santiago (PC). Departamento de Pediatría Hospital San Borja-Arriarán. Facultad de Medicina, Universidad de Chile, Santiago (CLA).

Los autores declaran no tener conflictos de interés.

Correspondencia a: ernesto.paya@vtr.net isabelnoemi@gmail.com rtassara@med.uchile.cl cavilesı@gmail.com 


\section{Profilaxis}

Son importantes las medidas no farmacológicas. Han de extremarse medidas higiénicas en el diario vivir, especialmente en el lavado de manos prolijo y en la preparación de alimentos. Otras precauciones relevantes son: evitar el contacto con mascotas, ingerir exclusivamente agua hervida, alimentos siempre bien cocidos, especialmente carnes y verduras, no consumir alimentos en lugares públicos, lavado prolijo de utensilios, separar áreas limpias y sucias en la preparación de los alimentos.

Se debe efectuar estudio serológico ( $\mathrm{IgG}$ ) del binomio D-R pre-trasplante, por técnica de RIFI o ELISA ${ }^{8}$.

En los receptores, deberá realizarse seguimiento periódico post-trasplante, con RPC desde los 15 días, una vez a la semana, por un mínimo de 6 meses y hasta tener CD4 $\geq 200$ céls $/ \mathrm{mm}^{3}$. Podrá prolongarse en presencia de EICH y en tratamiento inmunosupresor. La RPC negativa no descarta una reactivación, por lo que siempre debe prevalecer su expresión clínica. Después de tres meses post-trasplante, se sugiere complementar con serología ELISA-RIFI cada 15 días. La quimioprofilaxis anti-toxoplasma con cotrimoxazol ha demostrado ser efectiva para reducir la incidencia de la enfermedad en pacientes receptores de TPH y de trasplante corazón o corazón pulmón ${ }^{4,9}$. Los pacientes con menor riesgo de enfermedad no necesitan profilaxis específica contra $T$. gondii ${ }^{4,9}$.

\section{Alternativas de profilaxis}

\section{Adultos}

- Pirimetamina-sulfadiazina, 1 comprimido de pirimetamina (25 mg)/día más 1 comprimido de sulfadiazina (500 mg) cada $12 \mathrm{~h}$ acompañado de ácido folínico: $10-15 \mathrm{mg} / \mathrm{m}^{2}$ de superficie corporal, desde día 30 a 180 post-trasplante. Prolongar o reestablecer en EICH o inmunodeficiencia mantenida.

- Espiramicina: 25-50 mg/kg/día, con máximo 2-3 gr. Uso diario ${ }^{10}$.

- Otras alternativas a evaluar, con precaución, son clindamicina y azitromicina.

\section{Niños}

- Pirimetamina: 0,25 mg/kg/día y sulfadiazina $25 \mathrm{mg} /$ $\mathrm{kg} /$ día, uso diario, más ácido folínico $10-15 \mathrm{mg} / \mathrm{kg} / \mathrm{m}^{2}$.

- Espiramicina: $25-50 \mathrm{mg} / \mathrm{kg} /$ día, uso diario.
Duración de la profilaxis. En TPH: desde el implante hasta seis meses post trasplante como mínimo. Se recomienda extender hasta los 12 meses o más, sólo en circunstancias especiales, como EICH o inmunosupresión prolongada $^{4,9}$.

Reacciones adversas a la profilaxis. Se han descrito mielosupresión en el caso de cotrimoxazol, pirimetamina y sulfadiazina; diarrea por clindamicina, toxicidad hepática y pancreática por cotrimoxazol y espiramicina, exantema por cotrimoxazol, espiramicina, pirimetamina y sulfadiazina ${ }^{4,11-13}$.

Recomendación $N^{\circ}$ 6: Profilaxis de toxoplasmosis

- Medidas no farmacológicas: Lavado de manos e higiene en la manipulación de alimentos

- Seguimiento periódico post-trasplante con RPC desde los 15 días, una vez por semana durante seis meses

- Los pacientes con menor riesgo de enfermedad por T. gondii deben recibir profilaxis universal con cotrimoxazol y no requieren profilaxis específica contra T. gondii (A2)

- En los pacientes con mayor riesgo de enfermedad por T. gondii parece suficiente la profilaxis universal con cotrimoxazol. No obstante, en caso de no poder realizar ésta, debe establecerse profilaxis alternativa específica contra T. gondii (B3)

\section{Resumen}

Toxoplasmosis es una zoonosis ampliamente distribuida, producida por el parásito T. gondii. En Chile la seroprevalencia se ha estimado entre $20-37 \%$ en la población general. Se han definido grupos de riesgo de adquirir o reactivar la infección por $T$. gondii en pacientes sometidos a TOS y a TPH: trasplante cardíaco o de corazón-pulmón con D (+) y R (-); TPH alogénico con $\mathrm{R}(+)$; TPH con células de cordón; EICH activa; antecedentes de toxoplasmosis clínica previa y uso de corticoesteroides por tiempo prolongado o en altas dosis. De manera universal son importantes el lavado de manos e higiene en manipulación de alimentos y el seguimiento periódico post-trasplante con RPC desde los 15 días, una vez por semana, durante seis meses. Todos los pacientes con TOS y TPH, independiente de su riesgo, deben recibir profilaxis universal con cotrimoxazol y no requieren otra profilaxis específica contra T. gondii (A2 ). Es particularmente importante que en los pacientes de alto riesgo que no puedan recibir cotrimoxazol, se establezca profilaxis alternativa específica contra T. gondii (B3). 


\section{Referencias bibliográficas}

1.- Contreras M, Schenone H, Salinas P, Sandoval L, Rojas A, Villarroel F, et al. Seroepidemiology of human toxoplasmosis in Chile. Revista do Instituto de Medicina Tropical de Sao Paulo 1996; 38: 431-5.

2.- Abarca K, Vial P A, Zamorano J, Paris C, Ferres M, Villarroel L, et al. Seroprevalence of cytomegalovirus and Toxoplasma gondii in healthy subjects under 30 years old in Santiago, Chile. Rev Med Chile 1997; 125: 531-8.

3.- Zamorano C G, Contreras M C, Villalobos S, Sandoval L, Salinas P. Seroepidemiological survey of human toxoplasmosis in Osorno, Region X, Chile, 1998. Bol Chil Parasitol 1999; 54: 33-6.

4.- Derouin F, Pelloux H. Prevention of toxoplasmosis in transplant patients. Clin Microbiol Infect 2008; 14: 1089-101.
5.- Dykewicz C A, Jaffe H W, Kaplan J E, Spira T J, Jarvis W R, Edlin B R, et al. Guidelines for Preventing Opportunistic Infections Among Hematopoietic Stem Cell Transplant Recipients. MMWR Morbid Mortal Wkly Rep 2000;Vol. 49/No. RR-10: 1-127.

6.- Martino R, Cordonnier C. Toxoplasmosis following allogeneic hematopoietic stem cell transplantation. Bone Marrow Transplant 2003; 31: 617-8; author reply 9.

7.- de Medeiros B C, de Medeiros C R, Werner B, Loddo G, Pasquini R, Bleggi-Torres L F. Disseminated toxoplasmosis after bone marrow transplantation: report of 9 cases. Transpl Infect Dis 2001; 3: 24-8.

8.- Documento 13913. Laboratorio de referencia de parasitología. Instituto de Salud Pública. (Accessed 5 de septiembre 2011, 2011, at.

9.- Baden L R, Katz J T, Franck L, Tsang S,
Hall M, Rubin R H, et al. Successful toxoplasmosis prophylaxis after orthotopic cardiac transplantation with trimethoprimsulfamethoxazole. Transplantation 2003; 75: 339-43.

10.- Brook I. Pharmacodynamics and pharmacokinetics of spiramycin and their clinical significance. Clin Pharmacokin 1998; 34: 303-10.

11.- Parasitología Cd. Parte IV. Enfermedad de Chagas en pacientes inmunocomprometidos. Rev Chilena Infectol 2008; 25: 289-92.

12.- Bowden R, Ljungman P, D. S. Parasites after haematopoietic stem cell or solid organ transplantation. 3 ed; 2010.

13.- Apt W, Heitmann I, Jercic I, Jofré L, Muñoz P, Noemí I, et al. Guías clínicas de la enfermedad de Chagas 2006 : Parte I - VI Rev Chilena Infectol 2008; 25: 189-389. 